\title{
Analgesia Accompanying Food Consumption Requires Ingestion of Hedonic Foods
}

\author{
H. Foo ${ }^{1}$ and Peggy Mason ${ }^{1,2}$ \\ ${ }^{1}$ Department of Neurobiology and ${ }^{2}$ Committee on Neurobiology, University of Chicago, Chicago, Illinois 60637
}

\begin{abstract}
Animals eat rather than react to moderate pain. Here, we examined the behavioral, hedonic, and neural requirements for ingestion analgesia in ad libitum fed rats. Noxious heat-evoked withdrawals were similarly suppressed during self-initiated chocolate eating and ingestion of intraorally infused water, sucrose, or saccharin, demonstrating that ingestion analgesia does not require feeding motivation, self-initiated food procurement, sucrose, or calories. Rather, food hedonics is important because neither salt ingestion nor quinine rejection elicited analgesia. During quinine-induced nausea and lipopolysaccharide (LPS)-induced illness, conditions when chocolate eating was presumably less pleasurable, analgesia accompanying chocolate consumption was attenuated, yet analgesia during water ingestion was preserved in LPS-injected rats who showed enhanced palatability for water within this context. The dependence of ingestion analgesia on the positive hedonics of an ingestate was confirmed in rats with a conditioned taste aversion to sucrose: after paired exposure to sucrose and LPS, rats no longer showed analgesia during sucrose ingestion but continued to show analgesia during chocolate consumption. Eating pauses tended to occur less often and for shorter durations in the presence of ingestion analgesia than in its absence. Therefore, we propose that ingestion analgesia functions to defend eating from ending. Muscimol inactivation of the medullary raphe magnus blocked the analgesia normally observed during water ingestion, showing the involvement of brainstem endogenous pain inhibitory mechanisms in ingestion analgesia. Brainstem-mediated defense of the consumption of palatable foods may explain, at least in part, why overeating tasty foods is so irresistible even in the face of opposing cognitive and motivational forces.
\end{abstract}

\section{Introduction}

Animals innately seek pleasure and avoid pain. When motivational conflicts between these fundamental goals occur, an animal must either endure pain to attain pleasure or relinquish pleasure to avoid pain. Current evidence suggests that the former strategy predominates over the latter. In healthy rats, selfinitiated eating takes precedence over reacting to brief, moderately painful thermal stimulation, even in ad libitum fed animals (Foo and Mason, 2005). While eating paw-held food, rats either do not withdraw or withdraw at longer latencies from noxious paw heat. Similarly, food-deprived cats withdraw at longer latencies and vocalize less in response to brief noxious stimuli while eating than when not eating (Casey and Morrow, 1983). Pain behaviors, favoring and lifting of an arthritic leg, were almost completely eliminated when food-deprived arthritic chickens were given access to food (Wylie and Gentle, 1998). Eating also takes precedence over protective reactions to noxious stimuli in invertebrates, suggesting a phylogenetically conserved behavioral hierarchy in which feeding trumps reacting to pain (Davis et al., 1974a; Gillette et al., 2000). In the mollusk Pleurobranchaea, feeding takes precedence over withdrawing from pain, mating, and

Received July 21, 2009; revised Aug. 27, 2009; accepted Sept. 2, 2009.

This work was supported by National Institute on Drug Abuse and the Women's Council of the Brain Research Foundation. We thank Drs. Richard Bodnar, Aaron Fox, Kevin Hellman, Donald Katz, Malcolm Nason, RobertPerlman, Nino Ramirez, and Peter Redgrave for helpful comments and Nasya Mendoza-Elias for technical assistance.

Correspondence should be addressed to Peggy Mason, Department of Neurobiology, University of Chicago, 947 East 58th Street, Chicago, IL 60637. E-mail: p-mason@uchicago.edu.

DOI:10.1523/JNEUROSCI.3514-09.2009

Copyright $\odot 2009$ Society for Neuroscience ～0270-6474/09/2913053-10\$15.00/0 the righting reflex but is itself suppressed by egg laying (Davis et al., 1974a,b). This behavioral hierarchy provides adaptive advantage, ensuring that the mollusk feeds on infrequently available carrion but not on its own nutritious eggs.

A feeding bout can be divided into two phases: food procurement and food consumption. Food procurement requires the forebrain, whereas the brainstem is sufficient for rudimentary oral ingestive responses to innately palatable substances and for the oral and somatic rejection of unpalatable substances (Grill and Norgren, 1978a,b; Watts, 2000; Berthoud, 2002). Food choice, a component of procurement, is affected by hunger and food palatability: minimally palatable food will not be eaten by an animal unless the animal has been food deprived, whereas a sated animal will seek out only highly palatable food (Cabanac and Johnson, 1983). In contrast to procurement, food consumption is relatively independent of hunger and food preference: an animal that does not initiate feeding will ingest palatable substances that are directly infused into the mouth (Kaplan et al., 1990). Rats even ingest intraorally infused substances that are not preferred, that is, substances that they would normally choose not to drink, such as concentrated salt. Quinine, an innately avoided substance, evokes some ingestive reactions at very low concentrations but elicits rejection reactions, typified by gaping, at higher concentrations.

Here, we investigated the behavioral, hedonic, and neural requirements for pain suppression during ingestion in ad libitum fed rats. To determine whether self-initiated feeding and procurement of food are needed to trigger pain suppression during feeding, we tested for analgesia during ingestion of intraorally 
Table 1. Consumption of chow and chocolate chips

\begin{tabular}{|c|c|c|c|c|c|}
\hline & $\begin{array}{l}\text { Total occurrences } \\
\text { of eating chow }\end{array}$ & $\begin{array}{l}\text { Total number of } \\
\text { chips retrieved (offered) }\end{array}$ & $\begin{array}{l}\text { Chips dropped } \\
\text { (unretrieved) }\end{array}$ & $\begin{array}{l}\text { Average } \pm \text { SEM } \\
\text { chips eaten }\end{array}$ & $\begin{array}{l}\text { Total of offered } \\
\text { eaten (\% eaten) }\end{array}$ \\
\hline \multicolumn{6}{|l|}{ Experiment 1: infusate } \\
\hline Water $(n=16)$ & 1.0 & $46.5(46.5)$ & 5.0 & $2.9 \pm 0.2$ & \\
\hline Sucrose $(n=15)$ & 1.0 & $43.0(43.0)$ & 1.0 & $2.9 \pm 0.2$ & \\
\hline Saccharin $(n=12)$ & 2.0 & $33.0(33.0)$ & 1.0 & $2.8 \pm 0.3$ & \\
\hline Salt $(n=12)$ & 0.0 & $35.0(35.0)$ & 1.0 & $2.9 \pm 0.2$ & \\
\hline \multirow[t]{2}{*}{ Quinine $(n=13)$} & 1.0 & $38.5(38.5)$ & 3.0 & $3.0 \pm 0.2$ & \\
\hline & & & & & $196.0(100 \%)$ \\
\hline \multicolumn{6}{|l|}{ Experiment 2: nausea } \\
\hline Water $(n=14)$ & 2.0 & 71.5 (71.5) & 3.0 & $5.1 \pm 0.1$ & \\
\hline Salt $(n=16)$ & 1.0 & $81.0(81.0)$ & 2.0 & $5.1 \pm 0.1$ & \\
\hline \multirow{2}{*}{ Quinine ( $n=16)$} & 1.0 & $82.5(82.5)$ & 13.0 & $5.2 \pm 0.2$ & \\
\hline & & & & & $235.0(100 \%)$ \\
\hline \multicolumn{6}{|l|}{ Experiment 3: LPS } \\
\hline $\operatorname{LPS}(n=19)$ & 2.0 & $162.0(177.0)$ & $(15.0)$ & $8.5 \pm 0.8$ & $162.0(91.5 \%)$ \\
\hline \multirow[t]{2}{*}{ Saline $(n=14)$} & 6.0 & $141.0(141.0)$ & 0.0 & $10.1 \pm 0.5$ & $141.0(100 \%)$ \\
\hline & & & & & $303.0(95.3 \%)$ \\
\hline \multicolumn{6}{|l|}{ Experiment 4: CTA } \\
\hline Paired (conditioning, $n=19$ ) & 1.0 & $49.5(49.5)$ & 2.0 & $2.9 \pm 0.2$ & \\
\hline Unpaired (conditioning, $n=14$ ) & 1.0 & $420.0(42.0)$ & 0.0 & $3.0 \pm 0.1$ & \\
\hline Paired (test, $n=19$ ) & 4.0 & $46.0(46.0)$ & 0.0 & $2.7 \pm 0.2$ & \\
\hline \multirow[t]{2}{*}{ Unpaired (test, $n=14$ ) } & 4.0 & $41.0(41.0)$ & 0.0 & $2.9 \pm 0.1$ & \\
\hline & & & & & $178.5(100 \%)$ \\
\hline Grand total & 27.0 & $912.5(927.5)$ & $31.0(15.0)$ & & 912.5 (98.4\%) \\
\hline
\end{tabular}

During testing, rats ate freely available chow infrequently but ate almost every chip offered. There were no significant differences in the number of chips eaten between groups in any of the experiments. Chips were dropped very rarely (chips dropped), but, when this occurred, rats always picked the chip back up and resumed eating. Although some LPS-injected rats were hypophagic (unretrieved), they still retrieved and ate $>90 \%$ of chips offered. (TA, Conditioned taste aversion.

infused substances. Furthermore, by infusing different substances into the mouth, we examined whether the analgesia evident during feeding was dependent on the presence of sucrose, the nutritional value of the ingestate, or simply on the act of ingestion itself. The role of hedonics was examined by inducing states of nausea and illness and by changing the valence of sucrose by associating it with illness. Finally, we tested whether the medullary raphe magnus (RM), a critical contributor to endogenous analgesia (Fields et al., 1983; Gebhart, 2004) and analgesia evoked by self-initiated procurement and ingestion of chocolate chips (Foo and Mason, 2005), also mediates analgesia during intraorally induced ingestion of water that does not involve the first two phases of feeding.

\section{Materials and Methods}

Animals. A total of 220 adult male, Sprague Dawley rats (300-450 g; Charles River Laboratories) were used. Rats were housed in plastic cages in a vivarium maintained at $23-25^{\circ} \mathrm{C}$ and were given ad libitum access to chow and water. All procedures were reviewed and approved by the University of Chicago Institutional Animal Care and Use Committee.

Apparatus. The test cage was a Plexiglas box $(25 \times 45 \times 35 \mathrm{~cm})$ with a wire mesh floor. A modified Hargreaves apparatus (Hargreaves et al., 1988) was used to deliver radiant heat to the hindpaw. Heat intensity was set to elicit baseline withdrawal latencies of $\sim 3 \mathrm{~s}$, and the maximum stimulus lasted $8 \mathrm{~s}$. Cameras located in the front, side, and below the cage recorded the rat's behaviors, and a final one recorded a timer synchronized to electromyographic (EMG) data acquisition. All images were acquired simultaneously with an Everplex Quad Processor (Everfocus).

Intraoral infusions were made via a fluid swivel (Instech Laboratories) and an injector connected to the intraoral cannula. A pump (BAS) delivered intraoral infusions at a rate of $0.35 \mathrm{ml} / \mathrm{min}$.

Surgical preparation. Rats were anesthetized with pentobarbital (60 $\mathrm{mg} / \mathrm{kg}$, i.p.), placed in a stereotax, and maintained at $\sim 37^{\circ} \mathrm{C}$. An intraoral cannula was implanted (Grill and Norgren, 1978a,b). In a subset of animals $[n=16,12,14$, and 14 in intraoral infusion, nausea, lipopolysaccharide (LPS), and conditioned taste avoidance experiments, respectively], stainless steel wires (Cooner Wire) were sutured into the anterior digastric
(AD) and genioglossus muscles using a ventral approach (Travers and Norgren, 1986) and into the biceps femoris muscles for EMG recording. The EMG leads were attached to a microconnector (Omnetics) and affixed to the skull.

In a second subset of animals, the involvement of RM in producing analgesia during ingestion of intraoral water or salt was examined. An intraoral cannula and a 26 gauge stainless steel guide cannula (Plastics One) were implanted above the target nucleus (anteroposterior, -11.3 $\mathrm{mm}$; lateral, $0.0 \mathrm{~mm}$; ventral, $-7.5 \mathrm{~mm}$ relative to bregma). Both cannulae were cemented to the skull with dental acrylic and capped with dummy caps to maintain patency. All rats were allowed at least $7 \mathrm{~d}$ to recover from surgery.

Chocolate delivery. Before surgery, all rats, except those used in the microinjection study and in two-bottle preference tests, were trained to eat Nestlé Toll House milk chocolate chips for at least 2 weeks. Rats were habituated to the apparatus and exposed to noxious heat stimulation at least once to minimize any novelty effects during testing. They were preexposed to intraoral infusions of the infusates, except when a novel flavor, sucrose, was required to establish conditioned taste avoidance. All familiarization and testing sessions took place during the rat's diurnal cycle in a room maintained at $23-25^{\circ} \mathrm{C}$. To minimize any association of noxious paw heat with ingestion, infusions and a half or a full chocolate chip were occasionally given without accompanying paw heat.

During testing, chocolate chips were placed into the cage by the investigator (H.F.) one at a time, with an interval of at least $10 \mathrm{~min}$ between chips. When provided with chocolate, a rat usually retrieved the chip within several seconds, ambulated to a different part of the cage, and then sat on its haunches and ate the chip. Noxious heat was applied while the rat was eating the chip, after taking at least one bite. In LPS-injected rats in which the chip was not retrieved or was retrieved but not fully eaten ( 8 of 19), a second chip was given at least 10 min later. The numbers of chips offered, retrieved and eaten, and the instances of eating chow are shown in Table 1.

Intraoral infusions. Five groups of rats were tested for paw withdrawal and lick latencies during quiet waking, chocolate eating, and intraoral infusions of water $(n=16)$, sucrose $(0.3 \mathrm{M}, n=15)$, saccharin $(0.01 \mathrm{M}$, $n=12)$, salt $(0.3 \mathrm{M}, n=12)$, or quinine $(0.0003 \mathrm{M}, n=13)$. During each test session, noxious paw heat was applied when the rat was in quiet wake, 
eating a chocolate chip, or receiving an intraoral infusion. Minimum intervals of $5 \mathrm{~min}$ between paw heat trials and $10 \mathrm{~min}$ between chocolate chips were used. The order of the test trials was generated randomly on each test day. Infusions were given when the rats were awake, lasted at least $30 \mathrm{~s}$, and were terminated $5-10 \mathrm{~s}$ after heat offset. All subjects were tested with only one infusate for only one test session.

Two-bottle preference tests. A separate cohort of rats, of similar weights to those used above, were given two bottles filled with water and sucrose $(0.3 \mathrm{M})$, saccharin $(0.01 \mathrm{M})$, salt $(0.3 \mathrm{M})$, or quinine $(0.0003 \mathrm{M})$. Rats were housed individually and were fed ad libitum. The volumes of water and sucrose, saccharin, salt, or quinine consumed over a $12 \mathrm{~h}$ period (7:00 P.M. to 7:00 A.M.) were measured. The placements of the two bottles were counterbalanced across rats for each type of solution versus water.

Quinine-induced nausea. Three groups of rats were tested for withdrawal and lick reactions during quiet waking, chocolate eating, and chocolate eating immediately after an intraoral infusion of water $(n=$ $14)$, salt $(0.3 \mathrm{M}, n=16)$, or quinine $(0.0003 \mathrm{M}, n=16)$. Noxious heat to the hindpaw during quiet waking and chocolate eating were randomly assigned and separated by an interval of $\geq 5 \mathrm{~min}$. In postinfusion trials, intraoral infusion of water, salt, or quinine was delivered at $0.35 \mathrm{ml} / \mathrm{min}$ for $1 \mathrm{~min}$. Immediately after the infusion, rats were given a chocolate chip and tested for their reactions to paw heat as they were eating. After $\geq 10$ min, a second intraoral infusion was administered, and rats were given a chocolate chip and retested for reactions to paw heat during chocolate eating.

LPS-induced illness. Rats were injected with LPS (200 $\mu \mathrm{g} / \mathrm{kg}, 10 \mathrm{ml} / \mathrm{kg}$, i.p.; $n=19)$ or saline $(10 \mathrm{ml} / \mathrm{kg}$, i.p.; $n=14)$ and placed in the test cage. For the ensuing $6-8 \mathrm{~h}$, paw withdrawal and lick reactions to noxious heat during quiet wake, chocolate eating, and intraoral water infusions were tested hourly.

Conditioned taste avoidance. On day 1 ("conditioning"), rats were tested for paw withdrawal and lick latencies during quiet waking, chocolate eating, and intraoral infusions of sucrose $(0.3 \mathrm{M})$. At the end of the session, sucrose was infused for $1 \mathrm{~min}$ without heat stimulation. Rats remained in the cage for $\sim 5 \mathrm{~min}$ and then were injected with LPS (200 $\mu \mathrm{g} / \mathrm{kg}, 10 \mathrm{ml} / \mathrm{kg}$, i.p.; "paired"; $n=19$ ) or equivolume saline ("unpaired"; $n=14$ ). On day 2 , paired rats were injected in their home cages with saline, and unpaired rats received LPS to equate for drug and injection exposures. On day 5 ("test"), noxious heat was applied during quiet waking, chocolate eating, and intraoral infusions of water and sucrose. Testing during quiet waking and chocolate eating were interspersed randomly between testing during water and sucrose infusions. All testing trials with intraoral water infusions were conducted before those with intraoral sucrose infusions. After testing, rats were returned to their home cages and water deprived. On days 6 and 7, 30 min two-bottle preference tests were conducted to determine relative intakes of water and sucrose $(0.3 \mathrm{M})$, with bottle locations counterbalanced across days.

$R M$ inactivation. On the first test day, an internal microinjection cannula (33 gauge) extending $3 \mathrm{~mm}$ beyond the tip of the guide cannula was placed in the cannula guide and connected to a swivel and a Hamilton 1 $\mu l$ microsyringe via polyethylene PE20 tubing. Muscimol (50 ng in 250 $\mathrm{nl}$ ) or an equivalent volume of saline, in counterbalanced order, was delivered at a rate of $\sim 125 \mathrm{nl} / \mathrm{min}$. After the microinjection, rats were tested for their reactions to noxious radiant heat during quiet waking and intraoral infusion of water or salt $(0.3 \mathrm{M})$, with an interstimulus interval of $\sim 5 \mathrm{~min}$. The testing order for quiet waking and intraoral infusions was counterbalanced across animals. Rats also received intraoral infusions of water or salt without accompanying heat to minimize any association between intraoral infusions and noxious heat. On the second test day, rats received the reverse drug and were tested in the same quiet waking/ intraoral infusion order as used on day 1 . After the second test day, rats were killed and the brains were removed for histological verification of the injection site. A total of 18 rats ( $n=10$ for water, $n=8$ for salt) had histologically verified injections sites in RM (see Fig. $4 B$ ).

Latency and eating measurements. Paw withdrawal and lick latencies were calculated as the intervals between heat onset and paw withdrawal or lick from video recordings. The duration of eating pauses was determined from a comparison of video and EMG recordings when available and from video recordings alone in other cases. Calculations based on video and on EMG recordings were highly correlated $(r=0.90)$. Animals that did not withdraw were assigned the cutoff latency of $8 \mathrm{~s}$.

To ensure that the latency measurements were not influenced by experimenter bias, an independent observer, blind to the objectives of the study, measured the withdrawal latencies of randomly selected trials $(n=$ 150) from each experimental group. Latencies calculated by the investigator (H.F.) and by the blind observer were highly correlated ( $r=0.96$ for paw withdrawal latency and 0.89 for paw lick latency).

Data acquisition. A preamplifier conditioned the EMG signal and amplified it fivefold. The signal was further amplified 10,000-fold by a differential alternating current amplifier (A-M Systems) and acquired at $1-3 \mathrm{kHz}$ by a Power1401 (Cambridge Electronic Design). The EMG signal was rectified and smoothed using Spike2 acquisition software (Cambridge Electronic Design). The number of anterior digastric muscle activations per second during chocolate eating and intraoral infusions was calculated.

Statistics. Mean withdrawal latencies during quiet wake, infusions, and chocolate eating were calculated for each animal in each of the groups. A repeated-measures ANOVA followed by post hoc Student-NewmanKeuls (SNK) test was used to determine statistical significance between conditions (quiet wake, infusion, and chocolate) within each of the groups in the infusate, nausea, conditioning, and RM inactivation studies. A mixed-design ANOVA followed by post hoc SNK test was used to analyze between- and within-subject differences in the LPS study. Distributions of withdrawal latencies were tested for normality. In cases in which a normality test failed, a repeated-measures ANOVA on ranks was performed. In all analyses, the $\alpha$ level was set at 0.05 . All statistics were calculated with the SigmaStat software package (version 3.5; Systat Software).

\section{Results}

\section{Self-initiated feeding, sucrose, and calories are not required for analgesia associated with feeding}

To determine whether self-initiated feeding is required to trigger analgesia during ingestion, we compared reactions to noxious stimulation during self-initiated chocolate eating and ingestion initiated by fluid infusion directly into the oral cavity, hereafter referred to as experimenter-induced ingestion. Rats in all groups withdrew from noxious heat at longer latencies while they were eating chocolate chips than during quiet wake (Fig. $1 A, B)$. Rats infused with water withdrew at longer latencies during water ingestion than during quiet wake, demonstrating that suppression occurred independently of self-initiated feeding and food procurement. This result also demonstrates that ingestion analgesia does not require that the ingestate contain either sugar or calories.

Although water is calorically empty, it possesses a special significance so that animals are motivated to drink water specifically. Therefore, to determine whether sugar or calories in an ingestate other than water are required to trigger analgesia, we tested reactions to noxious stimulation during experimenterinduced ingestion of $0.3 \mathrm{M}$ sucrose or $0.01 \mathrm{M}$ saccharin. As above, rats withdrew from noxious heat at longer latencies as they were eating chocolate chips than during quiet wake. Furthermore, while ingesting either sucrose or saccharin, rats also withdrew from noxious heat at longer latencies than they did during quiet wake (Fig. 1A). Because ingestion of saccharin, a sugar-empty and calorically empty food, yielded similar results to sucrose, a calorically dense food, the suppression of reactions to pain during ingestion does not rely on either the sugar or caloric content of the ingestate.

The withdrawal latencies recorded while rats ingested water, sucrose, or saccharin were not significantly different from those during chocolate eating (Fig. $1 A$ ). Given this surprising result, we asked whether the ingestates differed in palatability. We mea- 
sured palatability using a previously established method that relates palatability to the pattern of oro-facial movements elicited by an ingestate: highly palatable substances elicit ingestive reactions and with decreasing palatability, rejection reactions, chiefly gaping, increasingly accompany ingestive ones (Grill and Norgren, 1978a; Roitman et al., 2005). Rats were videotaped from below to visualize oral movements indicative of ingestion or rejection, which are in turn reflective of the palatability of a substance. To confirm ingestive oro-facial movements and oral-facial movements indicative of rejection (gaping), EMG wires were implanted into the genioglossus and anterior digastric muscles of a subset of rats receiving intraoral infusions of water $(n=$ $4)$, sucrose $(n=3)$, saccharin $(n=2)$, salt $(n=3)$, or quinine $(n=4)$. In agreement with previously published work, we found that oral ingestive movements and gaping have easily distinguished EMG signatures (Travers and Norgren, 1986; Roitman et al., 2005). During ingestion, AD bursts continued for many seconds and were of a nearly constant magnitude, whereas during gaping, 1-2 s episodes of AD bursts followed a characteristic time course of increasing and then decreasing magnitude (Fig. $1 D, E$ ).

Chocolate elicited a greater frequency of AD bursts $(6.3 \mathrm{AD} / \mathrm{s})$ than did sucrose $(5.1 \mathrm{AD} / \mathrm{s})$ or saccharin $(5.2 \mathrm{AD} / \mathrm{s})$, which in turn elicited more frequent bursts than water $(4.4 \mathrm{AD} / \mathrm{s})$. In the absence of gaping movements, these differences suggest that chocolate is more palatable than sucrose and saccharin, which in turn are more palatable than water. Because withdrawal latencies recorded while rats ingested water, sucrose, or chocolate were not different, ingestion-associated analgesia does not appear to be graded by palatability.

Ingestion is necessary but not sufficient for eliciting analgesia associated with feeding

To determine whether ingestion is required to trigger analgesia associated with feeding, noxious paw heat was applied during quiet wake, chocolate eating, or intraoral infusion of quinine $(0.0003 \mathrm{M})$ at a concentration that rats predominantly reject (Grill and Norgren, 1978a). Quinine consistently elicited gapes (average of 6.7 gapes per infusion; 13 of 13 rats) indicative of rejection. During quinine infusions, rats withdrew at baseline latencies not different from those during quiet wake (Fig. 1 $A, C$ ). Because infusion of water, sucrose, or saccharin, all of which are ingested, elevate withdrawal latencies whereas quinine, a rejected substance, does not, ingestion appears required for analgesia associated with feeding.

To determine whether ingestion is sufficient to elicit analgesia, we tested the reactions to noxious stimulation of rats receiving intraoral infusions of salt at a concentration $(0.3 \mathrm{M})$ that rats ingested but which did not elicit any gapes, consistent with published reports (Grill and Norgren, 1978a,b). Rats withdrew at baseline latencies during salt ingestion (Fig. $1 \mathrm{~A}$ ), evidence that ingestion, although required, is insufficient on its own to elevate withdrawal latencies.

\section{Analgesia accompanies ingestion of water and} preferred substances

Because water, sucrose, and saccharin, all of which are ingested, trigger ingestion analgesia but salt, which is also ingested, does not, it appears that some quality common to water, sucrose, and saccharin is not shared by salt. The concentrations of sucrose $(0.3$ $\mathrm{M}, \sim 10 \%)$ and saccharin $(0.01 \mathrm{M}, \sim 0.18 \%)$ were chosen on the basis of published reports so that they were close to the maximally preferred concentrations (Corbit and Luschei, 1969; Smith, 2000), whereas the concentration of salt ( $0.3 \mathrm{M})$ was selected because it is reportedly less preferred than water (Bare, 1949; Grill and Norgren, 1978a; Flynn and Grill, 1988). Rats are well known to avoid ingesting $0.0003 \mathrm{M}$ quinine and find it aversive (Grill and Norgren, 1978a). To confirm these published reports, we recorded the amount consumed by naive rats of each ingestate and water using an overnight two-bottle test. Indeed, rats preferred sucrose or saccharin over water and water over salt and quinine (data not shown). These results suggest that ingestion of water or substances preferred over water are accompanied by analgesia, whereas no analgesia accompanies intraoral infusion of nonpreferred substances, regardless of whether they are ingested (salt) or rejected (quinine).

\section{Ingestion must occur within a positive gustatory context to elicit analgesia}

Rats willingly ingest the three substances that trigger analgesia (water, sucrose, and saccharin) but avoid the one substance whose ingestion does not trigger analgesia (salt). These results suggest that ingestion must be accompanied by some degree of positive hedonics to trigger analgesia. We therefore asked whether decreasing the hedonics associated with chocolate eating would attenuate or block ingestion analgesia. Because chocolate eating is less pleasurable during nausea (Garcia and Ervin, 1968; Welch, 1981), rats were tested for reactions to noxious stimulation during chocolate eating after an intraoral infusion of quinine, a nauseating substance (Kratz and Levitsky, 1978), or water. To ensure that quinine was acting as a nauseating agent and not just as a nonpreferred taste, we tested a third group of rats during 


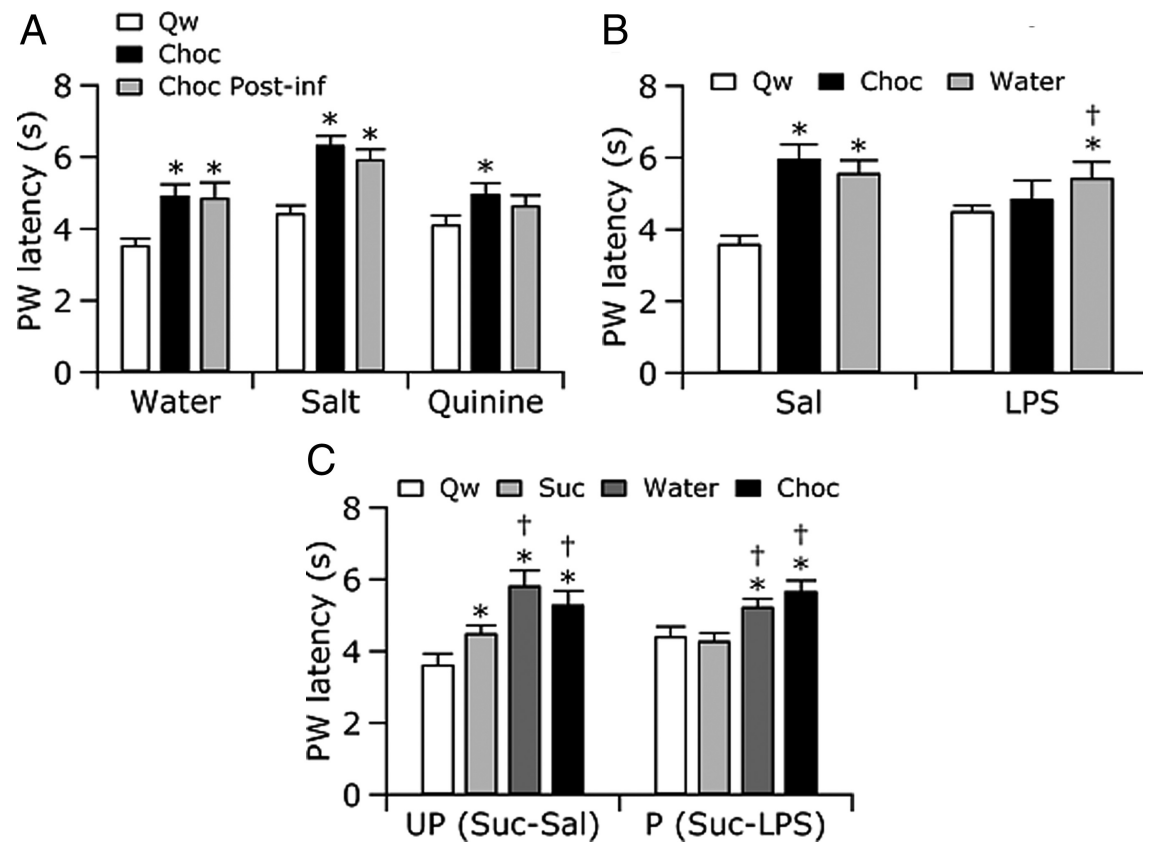

Figure 2. Suppression of heat-evoked paw withdrawals (PW) during ingestion was attenuated by nausea, illness, and conditioned taste avoidance. $A$, Rats withdrew at longer latencies ( \pm SEM) while eating chocolate (Choc) than during quiet wake ( $(\mathrm{w})$. Previous infusions had no effect on withdrawal latency during chocolate eating (Choc Post-inf) for animals receiving water (Water) or salt (Salt). However, when animals ate chocolate after quinine (Quinine), a nauseating substance, withdrawal latencies were not different from those during chocolate eating without a preceding infusion or those during quiet wake. B, Saline-injected rats (Sal) withdrew at longer latencies during chocolate (Choc) or water ingestion than during quiet wake, $2-4 \mathrm{~h}$ after injection. In contrast, LPS-injected rats (LPS) withdrew at longer latencies only during water ingestion, withdrawing at similar latencies during chocolate eating and quiet wake. C, Exposure to a cue previously paired with illness disengaged ingestion analgesia. Unpaired rats (UP) showed analgesia during chocolate and water ingestion and a partial analgesia during sucrose ingestion: withdrawal latencies during sucrose ingestion (Suc) were intermediate between chocolate/water ingestion and quiet wake (Qw). Paired rats (P) showed ingestion analgesia to chocolate and water ingestion but not to sucrose, withdrawing at similar latencies during sucrose infusion and quiet wake. ${ }^{*} p<0.05$ relative to $Q \mathrm{w}^{\dagger}{ }^{\dagger} p<0.05$ relative to without a preceding infusion $(\boldsymbol{A})$, chocolate eating $(\boldsymbol{B})$, or sucrose infusion (C); repeated-measures ANOVA followed by post hoc tests.

chocolate eating after infusions of salt, which, unlike quinine, does not evoke nausea. Although quinine-infused rats were likely nauseated, both they and salt-infused rats retrieved and ate all chips offered $(n=163.5)$ (Table 1$)$.

Withdrawal latencies during chocolate eating were unchanged by previous ingestion of water or salt (Fig. $2 \mathrm{~A}$ ). However, when eating chocolate after a quinine infusion, rats withdrew at latencies intermediate between those during chocolate eating without a preceding infusion and during quiet waking, evidence for attenuated eating analgesia during nausea. The attenuation of eating analgesia by nausea shows that food consumption during a period of compromised hedonics does not fully engage analgesia.

\section{Analgesia during ingestion requires positive hedonics specifically associated with the ingestate}

We considered that nauseated rats may have eaten chocolate to mask a lingering quinine taste within the mouth. To determine whether a negative experience unrelated to gustation alters ingestion analgesia, we asked whether rats with an immunological illness would show analgesia during ingestion. To this end, rats were injected with the endotoxin LPS, which acts on the immune system to produce sickness behaviors including hypolocomotion and fever (Dinarello et al., 1988; Rothwell, 1991). LPS-injected rats were unwell, showing piloerection and mild lethargy, but were still mobile and engaged in post-prandial grooming as often as saline-injected rats (data not shown). LPS- and saline- injected rats were tested for their reactions to noxious heat during quiet wake, intraoral water delivery, and chocolate eating for up to $8 \mathrm{~h}$ after injection. Although previous studies have reported that withdrawal latencies are shorter after LPS administration (Wiertelak et al., 1994), there were no significant differences between the average withdrawal latencies in saline- and LPS-injected rats during quiet wake at any time point after injection.

Illness after LPS administration is reported to evoke hypophagia (Plata-Salamán and Borkoski, 1993). Indeed, a minority of the LPS-injected rats (8 of 19, 42\%) failed to eat every chocolate chip offered, whereas all saline-injected rats ate every chip offered (Table 1). In total, six LPS-injected rats showed mild ( $<25 \%$ chips refused) and two moderate $(35-60 \%)$ hypophagia so that LPS-injected rats refused $8.5 \%$ of the chips offered.

During the expected peak of fever, 2-4 $\mathrm{h}$ after injection (Romanovsky et al., 1996), there was no evidence of analgesia associated with chocolate consumption because LPS-injected rats showed similar withdrawal latencies during quiet wake and chocolate eating (Fig. 2B). In contrast, sick rats withdrew at longer latencies when ingesting water. Associated with this water ingestion analgesia, the palatability of water increased, a result that confirms previous findings (Cross-Mellor et al., 2000). Specifically, LPS-injected rats reacted to water infusions with $5.4 \mathrm{AD} / \mathrm{s}$, a level of activation similar to that displayed by healthy non-injected rats receiving sucrose infusions (5.1 AD/s; see infusate experiment above). Interestingly, the motor reaction of LPS-injected rats to chocolate during fever was also $5.4 \mathrm{AD} / \mathrm{s}$, less than the reaction of healthy noninjected rats ( $6.3 \mathrm{AD} / \mathrm{s}$; see infusate experiment above). The augmentation of fever of water's palatability shows that rats valued water intake highly during febrile conditions. Furthermore, the apparent decline in the palatability of chocolate suggests that internally overheated rats may place a greater value on water than on calories, reminiscent of the preference for water over sugar solutions or energy-dense food demonstrated by rats in hot environments (Brobeck, 1960; Jacobs, 1971; Mogenson et al., 1971). Our findings that, during illness, water is increasingly palatable and evokes analgesia whereas eating energy-dense chocolate is less palatable and does not evoke analgesia suggest that analgesia during ingestion depends on positive hedonics specifically related to the ingested substance rather than on a generalized hedonic state.

\section{Ingestion analgesia is blocked when a good taste turns bad}

The persistence of analgesia during water ingestion in febrile rats suggests that sickness per se does not inhibit analgesia during ingestion and that a taste-specific negative association is necessary to switch off analgesia. To directly test whether a tastespecific negative association attenuates or blocks ingestion analgesia, we used a conditioned taste aversion paradigm to reverse the positive hedonics associated with sucrose. Rats in the 
paired condition were given intraoral sucrose infusions followed by LPS administration. These rats developed a conditioned taste avoidance to sucrose, which was confirmed in two-bottle intake preference tests of water and sucrose (data not shown). Rats in the unpaired condition were given intraoral sucrose infusions separate from LPS injections. These control rats showed a partial taste avoidance for sucrose (data not shown), likely stemming from an association between sucrose and the stress of the large volume $(\sim 4 \mathrm{ml}$, i.p. $)$ injection.

On the conditioning day, rats in both paired and unpaired groups showed withdrawal suppression during chocolate and sucrose ingestion compared with during quiet wake (data not shown). On the test day, both paired and unpaired rats showed withdrawal suppression during chocolate and water ingestion (Fig. 2C). However, paired rats withdrew at the same latencies during sucrose ingestion and quiet wake, evidence that ingestion analgesia was inhibited in these rats. During sucrose ingestion, unpaired rats withdrew at latencies intermediate between those during quiet wake and chocolate or water ingestion, consistent with a partial taste avoidance of sucrose and consequent attenuation of analgesia. A partial (unpaired group) or total (paired group) block of ingestion analgesia only occurred during ingestion of the conditioned stimulus, sucrose, and did not accompany ingestion of either water or chocolate.

Conditioning with sucrose-LPS did not change ingestive reactions to sucrose (5.4 AD/s on the test day compared with 5.1 $\mathrm{AD} / \mathrm{s}$ in on conditioning day; $t$ test, $p=0.60$ ), but there was a trend of an increase in rejection reactions to sucrose ( 1 of 19 rats gaped on the conditioning day and 5 of 19 on the test day; $\chi^{2}$ test, $p=0.08$ ), resulting in an overall decrease in the palatability associated with sucrose (Delamater et al., 1986; Berridge, 2000) and consequent inhibition of ingestion analgesia. Unpaired animals showed no changes in either ingestive movements $(5.3 \mathrm{AD} / \mathrm{s}$ on the test day compared with $5.8 \mathrm{AD} / \mathrm{s}$ on conditioning day; $t$ test, $p=0.30$ ) or rejection reactions ( 0 of 14 rats gaped on the conditioning day and 1 of 14 on the test day; $\chi^{2}$ test, $p=0.31$ ).

\section{Withdrawal latencies are not correlated to cumulative chip consumption}

Published work suggests that previous sucrose consumption has an analgesic effect on withdrawal latencies (Dum et al., 1983; Blass et al., 1987; Blass and Hoffmeyer, 1991; Segato et al., 1997). In the present study, there is no evidence that the analgesia observed was attributable to differences in the number of chocolate chips eaten. First, the average number of chips consumed by each rat was the same across groups within each experiment (Table 1). Furthermore, we found no relationship between the number of chips eaten and withdrawal latencies during quiet wake $\left(R^{2}=\right.$ $0.00-0.04)$ or during chocolate ingestion $\left(R^{2}=0.01-0.05\right)$ in rats tested with an infusate (Table 1, Experiment 1), in rats tested after water, quinine, or salt infusion (Table 1, Experiment 2), or in rats tested in the conditioned taste aversion paradigm (Table 1, Experiment 4).

In the LPS experiment, rats injected with LPS ate, on average, 8.5 chips, and saline-injected rats ate an average of 10.1 chips, a difference that was not significant. Nonetheless, to test whether the hypophagia exhibited by some LPS-injected rats (8 of 19) and consequent lower number of chips eaten could account for an attenuated analgesia attributable to cumulative chocolate ingestion, we performed a linear regression between the number of chips ingested and withdrawal latency. There was no relationship between the number of chips consumed and withdrawal latency during quiet wake in either saline- or LPS-injected rats $\left(R^{2}=\right.$ 0.01 and 0.01 , respectively), indicating that cumulative chocolate consumption did not produce an analgesic effect during quiet wake. Interestingly, withdrawal latency during chocolate consumption trended to decrease as cumulative chocolate consumption increased in LPS-injected rats $\left(R^{2}=0.07, p=0.06\right)$, whereas withdrawal latency during chocolate consumption increased as cumulative chocolate consumption increased in saline-injected rats $\left(R^{2}=0.10, p=0.05\right)$.

To determine whether the linear correlations observed could account for the analgesia during chocolate consumption observed in saline-treated but not LPS-injected rats, we compared observed data with that expected by a linear model. As an initial condition, we used the average withdrawal latency observed during quiet wake trials after consumption of one or two chocolates. Given the cumulative chocolate consumption of each trial, regression coefficients were then used to calculate expected latencies. Using this model, the average withdrawal latency expected during chocolate consumption in salineinjected rats was $3.9 \mathrm{~s}$, whereas the average observed was $5.8 \mathrm{~s}$. Similarly, the average withdrawal latency expected during chocolate consumption in LPS-injected rats was $3.6 \mathrm{~s}$, whereas the average observed was $5.1 \mathrm{~s}$. Thus, the linear correlations between chocolate consumption and withdrawal latency cannot account for the withdrawal latencies observed in either saline- or LPS-injected rats.

\section{Paw licks rarely occurred during chocolate eating}

During testing, rats $(n=213)$ procured and ate freely available chow very infrequently ( $n=27$ instances during 279 sessions), whereas all rats, except a minority of those injected with LPS ( 8 of $19)$, retrieved and ate every chocolate chip offered $(n=912.5)$ (Table 1). Once a rat, hypophagic or not, started to eat a chocolate chip, he finished it. These data demonstrate that rats who are mostly sated to chow readily retrieve and eat chocolate and show that chocolate is far more palatable than chow.

Whereas rats usually licked their paw after withdrawing from a noxious heat stimulus applied during quiet wake, rats who were eating chocolate rarely licked their paw (Fig. 3). During intraoral infusions, rats licked their paw at frequencies within the $95 \%$ confidence interval range observed during quiet wake, suggesting that rats were willing to lick their paw while ingesting infused fluids. However, because we did not record from muscles specifically engaged in swallowing, the extent to which ingestion was interrupted could not be determined.

\section{Analgesia during ingestion may function to defend food consumption from interruption}

The application of noxious heat stimulation caused rats to occasionally pause while eating, something that rarely happened when rats ate without stimulation. Pauses during eating chocolate were more frequent and longer after LPS injection, when analgesia was blocked, than after saline injection when analgesia was present (LPS, $86 \%, 7.0 \pm 3.1 \mathrm{~s}$; saline, $56 \%, 3.1 \pm 0.2 \mathrm{~s}$; $p$ values $<0.05)$, yet pauses did not lead rats to actually terminate eating bouts because rats consumed $>98 \%$ of the chips procured $(n=912.5)$ (Table 1). Although the defense of chocolate eating from ending may be attributable to the high palatability of chocolate, there were too few instances of chow eating to determine whether eating a less palatable food is less well defended. 
A

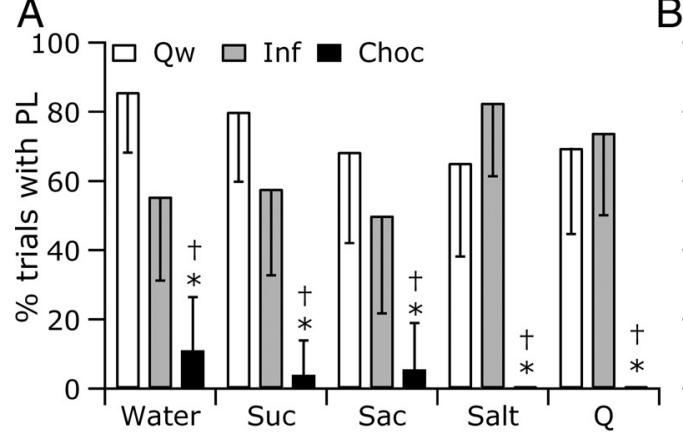

B

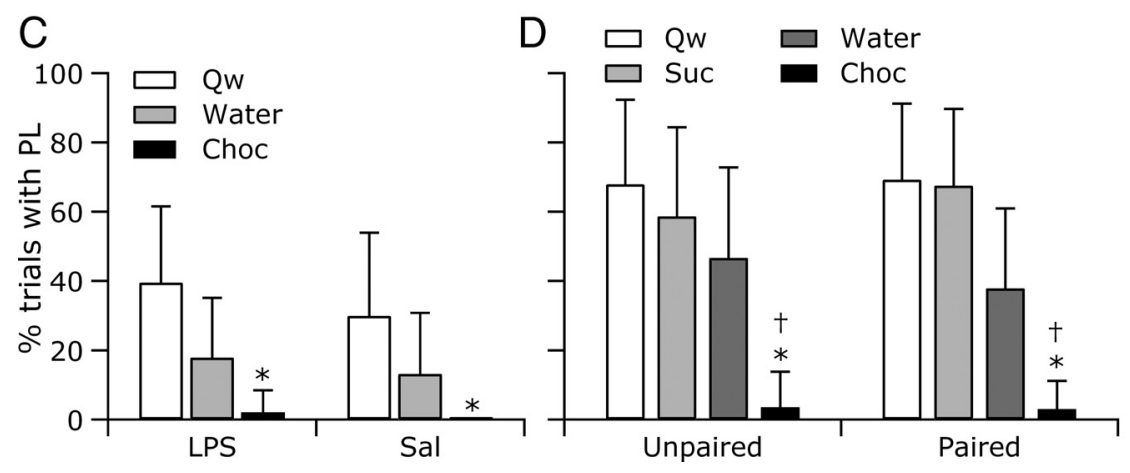

Figure 3. Paw licks (PL) were suppressed during chocolate eating but not during intraoral infusions. $A$, Rats typically licked their heated paw during quiet wake but not when eating chocolate. During intraoral infusions of both palatable and unpalatable substances, rats licked their paws in response to a noxious heat stimulus. $\boldsymbol{B}-\boldsymbol{D}$, When eating chocolate during quinine-induced nausea $(\boldsymbol{B})$, LPS illness $(\boldsymbol{C})$, or conditioned taste aversion to sucrose $(\boldsymbol{D})$, rats seldom licked their paw. Percentage of trials is outside the $95 \%$ confidence intervals for quiet wake (Qw; ${ }^{*}$ ) and infusion (Inf; $\dagger$ ). Conventions as in Figures 1 and 2.

\section{The medullary RM mediates the suppression of noxious heat-evoked withdrawals during ingestion of intraorally infused water}

Analgesia accompanying self-initiated eating is absent after inactivation of RM by muscimol microinjection (Foo and Mason, 2005). To determine whether RM also mediates the analgesia accompanying experimenter-induced ingestion, we tested the effect of saline or muscimol microinjection into RM on withdrawal reactions during quiet wake and ingestion evoked by intraoral infusion of water or salt. As shown in Figure $4 A$, microinjection of saline into RM had no effect on the increases in withdrawal latency during water ingestion. In contrast, after microinjection of muscimol into RM, rats withdrew at similar latencies during quiet wake and water ingestion, demonstrating that RM is critical to producing analgesia during ingestion even in the absence of self-generated initiation of feeding and food procurement. Withdrawal latencies were similar during quiet wake and salt ingestion, a finding that was unaltered by either saline or muscimol microinjection into RM, evidence that no active hyperalgesia accompanies salt ingestion.

\section{Discussion}

\section{Analgesia occurs concurrently with hedonic ingestion}

The current work establishes that suppression of pain reactions accompanies ingestion, independent of appetite, self-initiated feeding and food procurement, sucrose, sweetness, or calories. The analgesia accompanying water and saccharin ingestion in adult rats shows that ingestion analgesia does not require sugar, a sweet taste, or calories, providing very strong evidence that this analgesia is distinct from sucrose analgesia in neonates (Blass et al., 1987; Blass and Hoffmeyer, 1991; Blass and Shide, 1994; Ren et al., 1997). Because rats showed no analgesia while ingesting a salt solution, ingestion alone is not sufficient to elicit analgesia; rather, a positive hedonic value is also needed.

When tested subsequent to ingestion, palatable, non-sweet substances produce an analgesic effect in rat pups and adult humans. Specifically, 10-d-old rat pups that had received intraoral infusions of corn oil showed longer paw lift latencies than pups that had previously received intraoral infusions of water or had no infusions (Shide and Blass, 1989). In adult female humans, tolerance of pressure pain was higher among those who had previously drunk either a sweet soda or water, substances rated as equally palatable, than those who had had nothing to drink (Mercer and Holder, 1997). Although previous studies tested pain responses subsequent to ingestion, the present study shows a suppression of pain responses during ingestion, a clear demonstration that ingestion analgesia is not a post-ingestive effect.

When an animal tastes a fluid, it evaluates the fluid to make a palatability judgment and subsequently an ingestion "decision." In this scenario, it is entirely possible that this sensorimotor evaluation results in an expectation of reward, which itself triggers analgesia, even before the actual ingestion. In other words, tasting something good enough to ingest sets up a hedonic expectation, and this expectation, rather than the subsequent ingestion, elicits analgesia. Unfortunately, this possibility is not testable because it is impossible to prevent a rat from swallowing tasty intraoral fluids. Furthermore, tasting without either swallowing or rejecting is not a naturally occurring behavior in rodents or, indeed, in anyone. Nonetheless, the possibility exists that a brief exposure to a hedonic taste is sufficient to trigger the expectation of additional reward and that this expectation constitutes positive hedonics sufficient to trigger analgesia.

\section{Only foods with specific hedonic value elicit analgesia}

Decreasing the positive value of an ingestate, through either actual or a learned association with illness, attenuated ingestion analgesia. The attenuation of analgesia in nauseated and ill rats eating chocolate shows that negative affect, even when coupled with a highly palatable food such as chocolate, suppresses ingestion analgesia. However, the analgesia accompanying water ingestion in ill rats shows that, when the palatability of an ingestate-in this case water-is enhanced, ingestion analgesia occurs, even despite the concurrent negative experience of illness. Furthermore, the need for an ingestate to be both palatable and hedonically positive is confirmed by our demonstration that ingestion analgesia is blocked when a good taste, sucrose, turns bad via its association with illness.

\section{Is ingestion analgesia a form of pleasure analgesia?}

Pleasure-related analgesia is a term coined to refer to decreases in pain report and pain behaviors evoked by both rewarding stimuli, such as sucrose, and the expectation of such rewards (Dum et al., 1983; Dum and Herz, 1984; Reboucas et al., 2005; Leknes and Tracey, 2008). Whereas the analgesic conditions after days of 

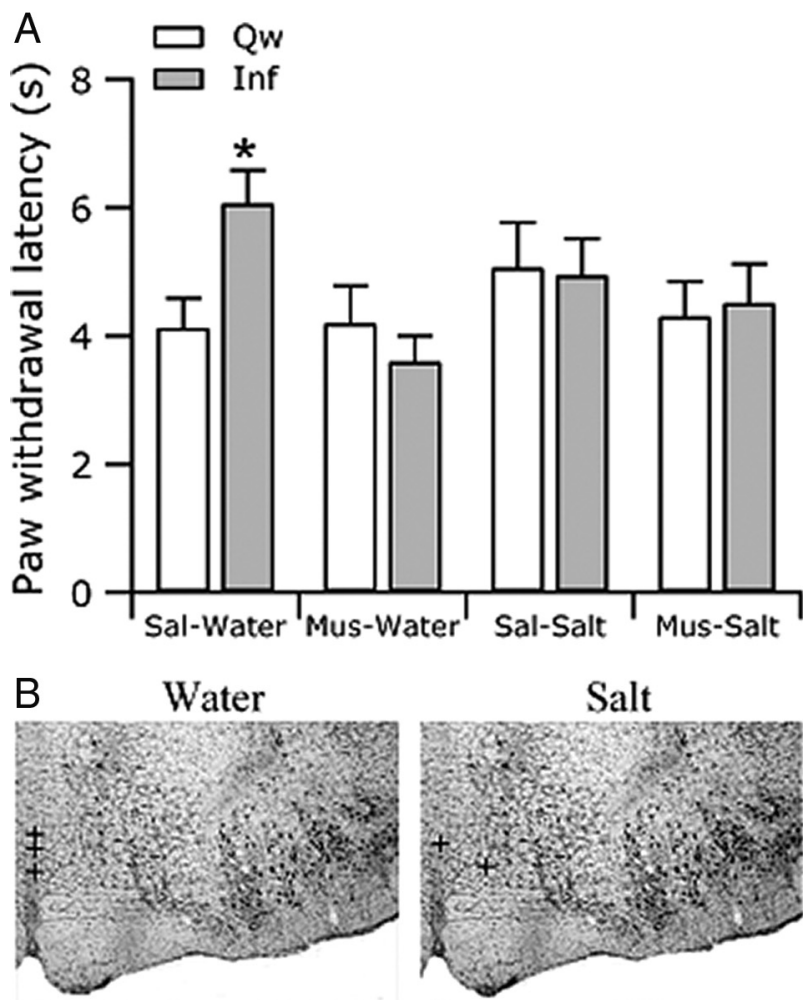

Bregma -11.0

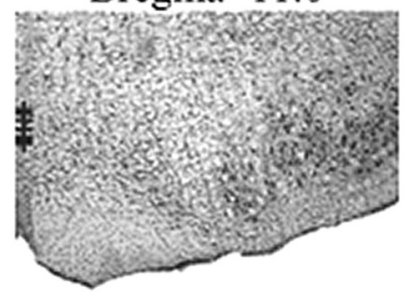

Bregma - 11.3
Bregma - 11.0

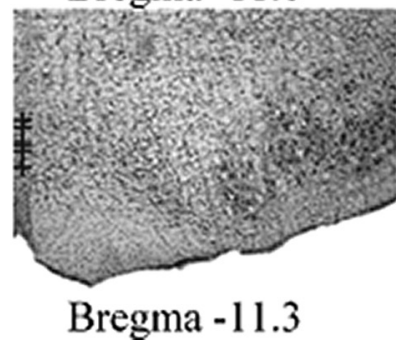

Figure 4. RM mediates the suppression of withdrawals associated with ingestion of intraorally infused water. $\boldsymbol{A}$, After saline (Sal) microinjection into RM, rats withdrew at longer latencies ( \pm SEM) during infusion (Inf) of water (Water) than during quiet wake (Qw). However, after muscimol (Mus) microinjection, the withdrawal latencies during water infusion and quiet wake were not different. Microinjection had no effect on withdrawal latencies during infusion of salt relative to during quiet wake. $\boldsymbol{B}$, All microinjections were made into RM on the midline.

sucrose ingestion or the expectation of receiving sucrose are tonic states (Dum et al., 1983; Dum and Herz, 1984; Reboucas et al., 2005), the analgesia accompanying ingestion demonstrated here and by others (see Introduction) is short lasting and phasic. In our studies, withdrawals were suppressed during ingestion but not during periods of quiet wake occurring before or after ingestion. Furthermore, there was no relationship between the number of chocolate chips ingested before a noxious stimulus and the withdrawal latency evoked by the stimulus. The finding that withdrawal suppression is time locked to ingestion is consistent with the finding that the firing pattern of RM cells is changed only during the ingestion component of an eating bout and not during either food retrieval or post-prandial grooming (Foo and Mason, 2005). Although it may seem intuitive that the pleasure of eating chocolate outweighs pain, it is less obvious that the pleasure of water ingestion outweighs pain in a fully hydrated animal. In fact, ad libitum fed rats were equally analgesic during the ingestion of chocolate and intraorally delivered water, sucrose, or saccharin.

Thus, ingestion analgesia requires ingestion, reflective of a threshold level of palatability, but is independent of appetite and food procurement.

\section{Sickness results in decreased food procurement but does not change food consumption}

Some LPS-sick rats were reluctant to work to procure chocolate but consistently completed eating chocolate once it had been retrieved. We hypothesize that, in the cases in which rats failed to retrieve chocolate, the effort needed for chip procurement, but not for ingestion, outweighed the estimated pleasure of chocolate consumption. These results predict that illness anorexia could be overcome by decreasing the cost of food procurement, as occurs when an ill person or animal is spoon fed. Although it may be counterproductive to divert energy from host defense to digestion, reducing the work required to procure food and increasing the motivation to eat by providing highly palatable food (Johnson and Cabanac, 1982) should stave off anorexia until an animal becomes too incapacitated to expend the energy needed for chewing, swallowing and the other mechanics of ingestion.

\section{Ingestion analgesia suppresses pauses in eating}

Shorter, less frequent pauses in eating occurred during conditions when withdrawal latency was elevated, suggesting that a function of eating analgesia is to protect eating from pauses and thus to serve as a defense against premature termination of an eating bout. However, eating analgesia did not completely defend food consumption from pauses as pauses, albeit brief ones, still occurred during eating analgesia. Furthermore, rats finished eating chocolate chips even when ingestion analgesia was attenuated or absent, evidence that ingestion analgesia cannot serve as the only defense of eating. Another contributing factor to the persistence of chocolate eating is likely the decrease in arousal that accompanies ingestion of palatable substances (Buchwald et al., 1964; Clemente et al., 1964; Hackett and Marczynski, 1969; Schwartzbaum et al., 1972).

Ingestion analgesia was less evident during ingestion of intraoral infusates than during chocolate consumption in the sense that paw licks were suppressed during the latter but not the former condition. The elimination of paw lick reactions during chocolate eating likely follows from the simple motor constraint that rats cannot lick their paw and eat chocolate simultaneously. Thus, another factor contributing to the defense of eating, as it occurs under natural conditions, is the constraint imposed by the motor plant, a single set of muscles, which cannot perform conflicting actions at the same time.

\section{RM mediates ingestion analgesia}

Since the dramatic demonstration that stimulation in the periaqueductal gray produces analgesia (Reynolds, 1969), a canonical pathway has been established: periaqueductal gray neurons project heavily to RM neurons that in turn modulate the nociceptive responses of dorsal horn cells (Basbaum and Fields, 1984; Sandkuhler, 2009). Pain-modulatory actions of RM neurons are critical for both the analgesic actions of narcotics and hyperalgesia consequent to nerve injury (Porreca et al., 2002; Gebhart, 2004). Although interest in the endogenous analgesia system has fueled the research efforts of many seeking efficacious, nonaddictive treatments for pain, the fundamental question of what intrinsic pain-modulatory pathways do for an animal living a normal life, who is neither in severe pain nor exposed to high levels of opioids, remains poorly understood. Here we demonstrate that RM mediates the suppression of reactions to painful 
stimuli during intraoral ingestion of water, suggesting a critical role for RM-mediated analgesia during eating, an activity that dominates everyday animal life.

Because RM is a region critical to opioid analgesia, its role in mediating analgesia during hedonic ingestion fits well with the contributions of opioids to food hedonics (Kelley et al., 2005). Opioid-mediated facilitation of palatability, together with the engagement of endogenous opioid analgesia during food consumption, would serve to ensure the uninterrupted ingestion of energy-dense foods, even in times of relative plenty. Furthermore, RM-mediated ingestion analgesia complements the proposed roles for RM in the analgesia accompanying sleep and micturition, additional activities critical to an animal's survival, which, like ingestion, are behaviors accompanied by some degree of disengagement from the world (Leung and Mason, 1998, 1999; Mason, 2001; Baez et al., 2005; Foo and Mason, 2005, Kelley et al., 2005). Thus, we propose that RM suppresses reactions to potentially distracting stimuli during everyday yet critical activities, thereby defending life-sustaining activities from interruption, a role befitting its location in the basal medulla.

Regions beyond RM are also likely involved in ingestion analgesia. In four animals in which the microinjection cannula was directed into reticular nuclei adjacent to RM, median paw withdrawal latencies were higher during water ingestion than during quiet wake after saline but not muscimol microinjection, suggesting that these regions also contribute to ingestion analgesia $(\mathrm{H}$. Foo, unpublished observations). This finding fits well with data showing similar anatomical and physiological properties of neurons throughout the ventromedial medulla as well as similar effects of stimulation throughout this region (for review, see Mason, 2001). Thus, it is likely that RM and neighboring regions together mediate ingestion analgesia. The additional involvement of regions more remote from RM cannot be precluded.

\section{$\mathrm{RM}$ is appropriately connected to receive information about the hedonic value of food}

Brainstem circuits can distinguish between substances that are innately ingested or rejected. However, when the valence of a food is changed, forebrain neurons in the nucleus accumbens code for the learned hedonic value of a food, which then determines whether a food is sought after or avoided (Roitman et al., 2005). Information from the nucleus accumbens reaches the hypothalamus, which projects both directly and indirectly, via the periaqueductal gray, to RM (Hosoya and Matsushita, 1981; Holstege, 1987; Sim and Joseph, 1991; Vertes and Crane, 1996; Hermann et al., 1997; Murphy et al., 1999). RM neurons project directly to the spinal cord with terminals concentrated in the superficial dorsal horn in which afferents coding for pain and temperature terminate (Basbaum and Fields, 1978, 1984). Because RM activation, by either chemical or electrical means, results in the suppression of somatosensory responses of medullary and spinal dorsal horns, descending pathways from RM could support ingestion analgesia.

\section{Ingestion analgesia may contribute to obesity in a world of readily available, energy-dense food}

Food consumption depends on brainstem circuitry that senses and reacts, by ingestion or rejection, to an intraoral substance (Grill and Norgren, 1978a,b). Because RM mediates pain suppression associated with ingestion, the brainstem supports both ingestion of readily available food and the defense of that ingestion from undue interruptions. In situations in which highly palatable food is readily available, the promotion by the brainstem of both food consumption and persistence to feed would work unopposed, resulting in hyperphagia and consequent obesity. Indeed, rats provided with an energy-dense cafeteria diet eat more, gain weight, and become obese (Rothwell and Stock, 1979). Humans also become hyperphagic when palatable food is readily available (Wansink, 1996; Wansink and Park, 2001; Painter et al., 2002; Diliberti et al., 2004; Wansink and Kim, 2005; Wansink et al., 2006), suggesting that tasty food within easy reach is destined for defended consumption in humans as well as other animals. Humans eat more when more food is available even when the food is stale or even when they are made cognizant of this tendency (Wansink and van Ittersum, 2007). The biological drive to consume palatable foods to completion outweighs opposing cognitive and motivational factors and is likely a major factor in the recent dramatic increase in obesity in modern human societies.

\section{References}

Baez MA, Brink TS, Mason P (2005) Roles for pain modulatory cells during micturition and continence. J Neurosci 25:384-394

Bare JK (1949) The specific hunger for sodium chloride in normal and adrenalectomized white rats. J Comp Physiol Psychol 42:242-253.

Basbaum AI, Fields HL (1978) Endogenous pain control mechanisms: review and hypothesis. Ann Neurol 4:451-462.

Basbaum AI, Fields HL (1984) Endogenous pain control systems: brainstem spinal pathways and endorphin circuitry. Annu Rev Neurosci 7:309-338.

Berridge KC (2000) Measuring hedonic impact in animals and infants: microstructure of affective taste reactivity patterns. Neurosci Biobehav Rev 24:173-198.

Berthoud HR (2002) Multiple neural systems controlling food intake and body weight. Neurosci Biobehav Rev 26:393-428.

Blass EM, Hoffmeyer LB (1991) Sucrose as an analgesic for newborn infants. Pediatrics 87:215-218.

Blass EM, Shide DJ (1994) Some comparisons among the calming and painrelieving effects of sucrose, glucose, fructose and lactose in infant rats. Chem Senses 19:239-249.

Blass E, Fitzgerald E, Kehoe P (1987) Interactions between sucrose, pain and isolation distress. Pharmacol Biochem Behav 26:483-489.

Brobeck JR (1960) Food and temperature. Recent Prog Horm Res 16:439-466.

Buchwald NA, Horvath FE, Wyers EJ, Wakefield C (1964) Electroencephalogram rhythms correlated with milk reinforcement In cats. Nature 201:830-831

Cabanac M, Johnson KG (1983) Analysis of a conflict between palatability and cold exposure in rats. Physiol Behav 31:249-253.

Casey KL, Morrow TJ (1983) Nocifensive responses to cutaneous thermal stimuli in the cat: stimulus-response profiles, latencies, and afferent activity. J Neurophysiol 50:1497-1515.

Clemente CD, Sterman MB, Wyrwicka W (1964) Post-reinforcement EEG synchronization during alimentary behavior. Electroencephalogr Clin Neurophysiol 16:355-365.

Corbit JD, Luschei ES (1969) Invariance of the rats rate of drinking. J Comp Physiol Psychol 69:119-125.

Cross-Mellor SK, Kent WD, Kavaliers M, Ossenkopp KP (2000) Examining the effects of lipopolysaccharide and cholecystokinin on water ingestion: comparing intake and palatability. Brain Res 861:220-232.

Davis WJ, Mpitsos GJ, Pinneo JM (1974a) The behavioral hierarchy of the mollusk Pleurobranchaea. I. The dominant position of the feeding behavior. J Comp Physiol 90:207-224.

Davis WJ, Mpitsos GJ, Pinneo JM (1974b) The behavioral hierarchy of the mollusk Pleurobranchaea. II. Hormonal suppression of feeding associated with egg-laying. J Comp Physiol 90:225-243.

Delamater AR, LoLordo VM, Berridge KC (1986) Control of fluid palatability by exteroceptive Pavlovian signals. J Exp Psychol Anim Behav Process 12:143-152.

Diliberti N, Bordi PL, Conklin MT, Roe LS, Rolls BJ (2004) Increased portion size leads to increased energy intake in a restaurant meal. Obes Res 12:562-568.

Dinarello CA, Cannon JG, Wolff SM (1988) New concepts on the pathogenesis of fever. Rev Infect Dis 10:168-189.

Dum J, Herz A (1984) Endorphinergic modulation of neural reward sys- 
tems indicated by behavioral changes. Pharmacol Biochem Behav 21:259-266.

Dum J, Gramsch C, Herz A (1983) Activation of hypothalamic betaendorphin pools by reward induced by highly palatable food. Pharmacol Biochem Behav 18:443-447.

Fields HL, Bry J, Hentall I, Zorman G (1983) The activity of neurons in the rostral medulla of the rat during withdrawal from noxious heat. J Neurosci 3:2545-2552.

Flynn FW, Grill HJ (1988) Effects of concentration presentation order and intraoral delivery on sucrose intake. Behav Neurosci 102:992-994.

Foo H, Mason P (2005) Sensory suppression during feeding. Proc Natl Acad Sci U S A 102:16865-16869.

Garcia J, Ervin FR (1968) Appetites, aversions, and addictions: a model for visceral memory. Recent Adv Biol Psychiatry 10:284-293.

Gebhart GF (2004) Descending modulation of pain. Neurosci Biobehav Rev 27:729-737.

Gillette R, Huang RC, Hatcher N, Moroz LL (2000) Cost-benefit analysis potential in feeding behavior of a predatory snail by integration of hunger, taste, and pain. Proc Natl Acad Sci U S A 97:3585-3590.

Grill HJ, Norgren R (1978a) The taste reactivity test. I. Mimetic responses to gustatory stimuli in neurologically normal rats. Brain Res 143:263-279.

Grill HJ, Norgren R (1978b) The taste reactivity test. II. Mimetic responses to gustatory stimuli in chronic thalamic and chronic decerebrate rats. Brain Res 143:281-297.

Hackett JT, Marczynski TJ (1969) Postreinforcement electrocortical synchronization and enhancement of cortical photic evoked potentials during instrumentally conditioned appetitive behavior in the cat. Brain Res 15:447-464.

Hargreaves K, Dubner R, Brown F, Flores C, Joris J (1988) A new and sensitive method for measuring thermal nociception in cutaneous hyperalgesia. Pain 32:77-88.

Hermann DM, Luppi PH, Peyron C, Hinckel P, Jouvet M (1997) Afferent projections to the rat nuclei raphe magnus, raphe pallidus and reticularis gigantocellularis pars alpha demonstrated by iontophoretic application of choleratoxin (subunit b). J Chem Neuroanat 13:1-21.

Holstege G (1987) Some anatomical observations on the projections from the hypothalamus to brainstem and spinal cord: an HRP and autoradiographic tracing study in the cat. J Comp Neurol 260:98-126.

Hosoya Y, Matsushita M (1981) Brainstem projections from the lateral hypothalamic area in the rat, as studied with autoradiography. Neurosci Lett 24:111-116.

Jacobs HL (1971) Ambient temperature, insulin, and available food choice as determinants of appetite. J Physiol (Paris) 63:291-292.

Johnson KG, Cabanac M (1982) Homeostatic competition in rats fed at varying distances from a thermoneutral refuge. Physiol Behav 29: 715-720.

Kaplan JM, Spector AC, Grill HJ (1990) Ingestion rate as an independent variable in the behavioral analysis of satiation. Am J Physiol 258: R662-R671.

Kelley AE, Baldo BA, Pratt WE, Will MJ (2005) Corticostriatalhypothalamic circuitry and food motivation: integration of energy, action and reward. Physiol Behav 86:773-795.

Kratz CM, Levitsky DA (1978) Post-ingestive effects of quinine on intake of nutritive and non-nutritive substances. Physiol Behav 21:851-854.

Leknes S, Tracey I (2008) A common neurobiology for pain and pleasure. Nat Rev Neurosci 9:314-320.

Leung CG, Mason P (1998) Physiological survey of medullary raphe and magnocellular reticular neurons in the anesthetized rat. J Neurophysiol 80:1630-1646.

Leung CG, Mason P (1999) Physiological properties of raphe magnus neurons during sleep and waking. J Neurophysiol 81:584-595.

Mason P (2001) Contributions of the medullary raphe and ventromedial reticular region to pain modulation and other homeostatic functions. Annu Rev Neurosci 24:737-777.

Mercer ME, Holder MD (1997) Antinociceptive effects of palatable sweet ingesta on human responsivity to pressure pain. Physiol Behav 61: 311-318.

Mogenson GJ, Mok A, Grace JE, Stevenson JA (1971) Behavioral and alimentary response of the rat to acute and chronic changes in environmental temperature. J Physiol (Paris) 63:346-349.
Murphy AZ, Rizvi TA, Ennis M, Shipley MT (1999) The organization of preoptic-medullary circuits in the male rat: evidence for interconnectivity of neural structures involved in reproductive behavior, antinociception and cardiovascular regulation. Neuroscience 91:1103-1116.

Painter JE, Wansink B, Hieggelke JB (2002) How visibility and convenience influence candy consumption. Appetite 38:237-238.

Plata-Salamán CR, Borkoski JP (1993) Centrally administered bacterial lipopolysaccharide depresses feeding in rats. Pharmacol Biochem Behav 46:787-791.

Porreca F, Ossipov MH, Gebhart GF (2002) Chronic pain and medullary descending facilitation. Trends Neurosci 25:319-325.

Rebouças EC, Segato EN, Kishi R, Freitas RL, Savoldi M, Morato S, Coimbra NC (2005) Effect of the blockade of $\mu_{1}$-opioid and $5 \mathrm{HT}_{2 \mathrm{~A}}$-serotonergic/ $\mathrm{a}_{1}$-noradrenergic receptors on sweet-substance-induced analgesia. Psychopharmacology 179:349-355.

Ren K, Blass EM, Zhou Q, Dubner R (1997) Suckling and sucrose ingestion suppress persistent hyperalgesia and spinal Fos expression after forepaw inflammation in infant rats. Proc Natl Acad Sci U S A 94:1471-1475.

Reynolds DV (1969) Surgery in the rat during electrical analgesia induced by focal brain stimulation. Science 164:444-445.

Roitman MF, Wheeler RA, Carelli RM (2005) Nucleus accumbens neurons are innately tuned for rewarding and aversive taste stimuli, encode their predictors, and are linked to motor output. Neuron 45:587-597.

Romanovsky AA, Kulchitsky VA, Akulich NV, Koulchitsky SV, Simons CT, Sessler DI, Gourine VN (1996) First and second phases of biphasic fever: two sequential stages of the sickness syndrome? Am J Physiol 271: R244-R253.

Rothwell NJ (1991) Functions and mechanisms of interleukin 1 in the brain. Trends Pharmacol Sci 12:430-436.

Rothwell NJ, Stock MJ (1979) Regulation of energy balance in two models of reversible obesity in the rat. J Comp Physiol Psychol 93:1024-1034.

Sandkühler J (2009) Models and mechanisms of hyperalgesia and allodynia. Physiol Rev 89:707-758.

Schwartzbaum JS, Kreinick CJ, Mello WF (1972) Cortical evoked potentials and synchronization of electrocortical activity during consummatory behavior in rats. Brain Res 36:171-182.

Segato FN, Castro-Souza C, Segato EN, Morato S, Coimbra NC (1997) Sucrose ingestion causes opioid analgesia. Braz J Med Biol Res 30:981-984

Shide DJ, Blass EM (1989) Opioidlike effects of intraoral infusions of corn oil and polycose on stress reactions in 10-day-old rats. Behav Neurosci 103:1168-1175.

Sim LJ, Joseph SA (1991) Arcuate nucleus projections to brainstem regions which modulate nociception. J Chem Neuroanat 4:97-109.

Smith JC (2000) Microstructure of the rat's intake of food, sucrose and saccharin in 24-hour tests. Neurosci Biobehav Rev 24:199-212.

Travers JB, Norgren R (1986) Electromyographic analysis of the ingestion and rejection of sapid stimuli in the rat. Behav Neurosci 100:544-555.

Vertes RP, Crane AM (1996) Descending projections of the posterior nucleus of the hypothalamus: Phaseolus vulgaris leucoagglutinin analysis in the rat. J Comp Neurol 374:607-631.

Wansink B (1996) Can package size accelerate usage volume? J Mark 60:1-14.

Wansink B, Kim J (2005) Bad popcorn in big buckets: portion size can influence intake as much as taste. J Nutr Educ Behav 37:242-245.

Wansink B, Park SB (2001) At the movies: how external cues and perceived taste impact consumption volume. Food Qual Pref 12:69-74.

Wansink B, van Ittersum K (2007) Portion size me: downsizing our consumption norms. J Am Diet Assoc 107:1103-1106.

Wansink B, van Ittersum K, Painter JE (2006) Ice cream illusions. Am J Prev Med 31:240-243.

Watts AG (2000) Understanding the neural control of ingestive behaviors: helping to separate cause from effect with dehydration-associated anorexia. Horm Behav 37:261-283.

Welch D (1981) Nutritional compromise in radiation therapy patients experiencing treatment-related emesis. J Parenter Enteral Nutr 5:57-60.

Wiertelak EP, Smith KP, Furness L, Mooney-Heiberger K, Mayr T, Maier SF, Watkins LR (1994) Acute and conditioned hyperalgesic responses to illness. Pain 56:227-234.

Wylie LM, Gentle MJ (1998) Feeding-induced tonic pain suppression in the chicken: reversal by naloxone. Physiol Behav 64:27-30. 